\title{
BMJ Open Comparison of open-skill and closed- skill exercises in improving the response inhibitory ability of the elderly: a protocol for a randomised controlled clinical trial
}

\author{
Liu Ke, ${ }^{1}$ Zhang Lanlan, ${ }^{2}$ Zhang Jian, ${ }^{3}$ Wei Jianing (D) ${ }^{3}$
}

To cite: Ke L, Lanlan Z, Jian Z, et al. Comparison of open-skill and closed-skill exercises in improving the response inhibitory ability of the elderly: a protocol for a randomised controlled clinical trial. BMJ Open 2021;11:e051966. doi:10.1136/ bmjopen-2021-051966

- Prepublication history for this paper is available online. To view these files, please visit the journal online (http://dx.doi. org/10.1136/bmjopen-2021051966).

LK and ZL are joint first authors.

Received 03 April 2021 Accepted 22 0ctober 2021

A) Check for updates

(c) Author(s) (or their employer(s)) 2021. Re-use permitted under CC BY-NC. No commercial re-use. See rights and permissions. Published by BMJ.

${ }^{1}$ Shanghai Punan Hospital, Shanghai, China

${ }^{2}$ School of Leisure Sport and Management, Guangzhou Sport University, Guangzhou, China

${ }^{3}$ School of Psychology, Shanghai University of Sport, Shanghai, China

Correspondence to

Wei Jianing;

2011916006@sus.edu.cn

\section{ABSTRACT}

Introduction As people age, they are more likely to experience a decline in their response inhibition ability, which interferes with daily life. Previous studies have shown that exercise intervention can improve the cognitive ability of the elderly, but research on open-skill and closed-skill exercises to improve the response inhibition in this age group is still limited. This study will explore the advantages of long-term intervention of table tennis (openskill exercise) compared with fit aerobics (closed-skill exercise) on the response inhibitory ability of the elderly. Methods and analysis A single-blind randomised controlled trial will be conducted. A total of 90 elderly subjects will be recruited and allocated randomly to the following groups: table tennis, fit aerobics and control. The interventions for the table tennis group and the fit aerobics group will be implemented in three 60 min sessions per week for 6 months; the control group will receive no exercise intervention. The primary assessment will be behavioural indicators of response inhibitory ability in the elderly based on the stop signal task. The secondary outcomes will include cognitive ability, mental status and depression in the elderly. Assessments will be conducted at baseline, 3 months, 6 months and 12 months.

Ethics and dissemination This study was approved by the Shanghai University of Sport Research Ethics Committee (102772019RT012) and will provide reference for the advantages of table tennis compared with other types of sports in improving the response inhibitory ability of the elderly. The results of this study will provide a theoretical basis for choosing the best exercise programme to improve the response inhibitory ability of the elderly.

Trial registration number This study has been registered prospectively in the Chinese Clinical Trial Registry (ChiCTR2100043616, 23 February 2021).

\section{INTRODUCTION}

Response inhibition ability is a type of cognitive control ability that can achieve behavioural goals by adjusting perception and motor function in the changing environment as well as be used to solve conflicts. ${ }^{1}$ As people
Strengths and limitations of this study

Because it is a sports intervention, the safety of the elderly during exercise is worth worrying about.

- How to select and control participants in a longitudinal study is one of the difficulties of this project.

- The patients will come from one geographic area, which limits the generalisability.

age, they are likely to experience a decline in their response inhibition ability, ${ }^{2-5}$ which can interfere with daily life and even the ability to live independently. Although some studies have shown that exercise intervention can improve the cognitive ability of the elderly, ${ }^{6}$ there are few studies on the effect of exercise intervention on reactive inhibition ability. In particular, research on the different types of exercise to improve the response inhibition ability of the elderly is still limited.

Sports can be divided into closed skill activities and open skill activities. ${ }^{7}$ The operating environment of closed-skill exercises, such as fit aerobics, fitness running, is stable; and the operating state is determined by the operator. In contrast, the operating environment of open-skill exercises, including those involved in the sports of table tennis, basketball, is unstable; and the operation state is determined by external conditions, such as a ball and opponents. In open-skill exercises, the participant must continuously adjust their behaviour to unpredictable stimuli and must invest more cognitive resources in the decision-making process. Previous studies have shown that open-skill exercises require more regulating capacity than closed-skill exercises. ${ }^{8}$ First, a study comparing the cognitive control of sports involving open-skill exercises with that of closed-skill exercises has revealed that athletes engaging in open-skill 
exercises have an advantage in cognitive control. It has been demonstrated that intervention with long-term open-skill exercise can improve cognitive function better than closed-skill exercise, ${ }^{7}$ but whether it can improve response inhibitory ability better is not clear.

As one of the classical paradigms to test response inhibition, the stop signal task (SST) tests the complete suppression of an ongoing action. The SST instructs the participant to perform a response when the go signal appears, but after a few go signals appear, a stop signal will appear at a specific time interval, and the individual will be asked to stop the response as far as possible. ${ }^{9}$ The strengths of this test are that a stop signal to evoke controlling behaviour is administered after a go signal is used, and the interval between these two signals dynamically changes regularly. ${ }^{10}$ In addition, the SST is capable of quantising inhibitory control behaviour. However, no response occurs when the participant holds up successfully. The stop signal response time (SSRT) is a more sensitive indicator to evaluate the response inhibitory ability. ${ }^{11}$ In short, to calculate the SSRT, all go-reactions (ie, go-trials in which the response was missed) are rankordered and assigned the maximum response time in order to compensate for the lacking response. Afterwards, the most recent stop signal delay (SSD) is subtracted from the response time corresponding to the stop-signal response percentile. The resulting value is termed the SSRT. ${ }^{12} 13$ These results can be used as an important index to evaluate the response inhibitory ability.

The proposed randomised clinical trial will examine the effects of table tennis (open-skill exercise) and fit aerobics (closed-skill exercise) on the primary outcome (behavioural task) of response inhibitory ability as well as on the secondary outcomes of cognitive ability, mental status and depression in the elderly. We hypothesised that different types of exercise intervention can affect the response inhibitory ability to varying degrees and that the effect of open-skill exercises is better.

\section{METHODS \\ Study design}

This study protocol of a study is designed as a prospective, single-blind randomised controlled trial (RCT). Eligible participants will be randomised into table tennis, fit aerobics, or control groups at a 1:1:1 ratio. The duration of exercise intervention will be 6 months. The cognitive function test (questionnaire and behavioural task) will be conducted before the intervention as well as at 3 months, 6 months and 12 months after the intervention. Participants who meet the criteria will undergo baseline assessments. All evaluations will be done at baseline and immediately after the exercise intervention (see figure 1 ). We will recruit healthy right-handed elderly participants with normal or corrected-to-normal vision and without a history of neurological diseases in the same way. The study is scheduled to begin in August 2021 and continue until August 2023.

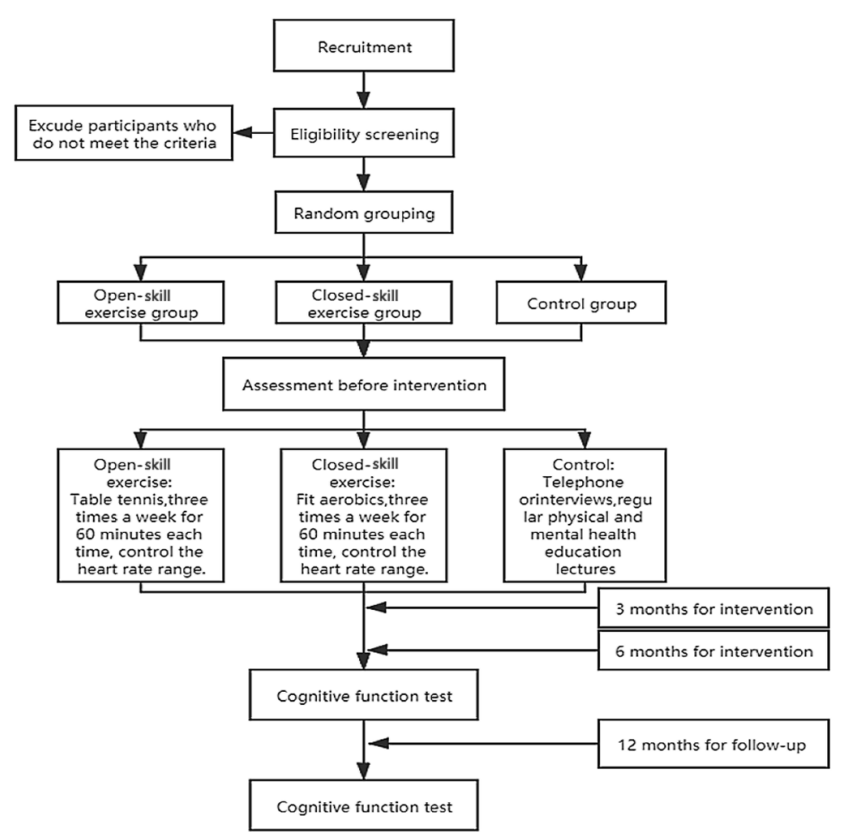

Figure 1 Flow diagram of the study design.

\section{Participants}

Any participants with heart disease, neurological disorder, musculoskeletal disorders, and other conditions not suitable for physical activity, contraindications to fMRI or transcranial magnetic stimulation (TMS) will be excluded. In addition, those with a regular exercise routine will be excluded. All participants will be between the ages of 60 and 70 years old and right-handed as assessed by the Edinburgh Handedness Inventory. Moreover, they must have their own residence.

Before and after the intervention, all groups will have their demographics statistically analysed for factors such as health status, physical fitness and cognitive function (table 1). Intergroup differences will be minimised before the intervention.

\section{Sample size}

From our previous studies on the modulation of SST performance, ${ }^{1415}$ we expected an effect of $\mathrm{r}=0.4$. Together with an $\alpha$-value of 0.05 and a power of $1-\beta=0.9$, a sample of at least 72 participants (24/group) was planned to find a similar effect. Calculations were carried out using G*Power, V.3.1 ${ }^{16}$ with anticipation of a $20 \%$ attrition rate. Therefore, we plan to recruit 90 subjects (30/group).

\section{Randomisation}

Eligible participants will be randomised into table tennis, fit aerobics or control groups at a 1:1:1 ratio after consenting and baseline assessment. We will use the Excel software to code the subjects in 1-90 according to the recruitment time, and then use the formula '=RAND ()' to generate the corresponding random sequence. By sorting the random sequence and then grouping it, 90 subjects will be randomly grouped. The above tasks will be completed by professional computer workers after the 
Table 1 Demographic and clinical characteristics of the study participants

\begin{tabular}{llll}
\hline & $\begin{array}{l}\text { Open } \\
\text { group }\end{array}$ & $\begin{array}{l}\text { Closed } \\
\text { group }\end{array}$ & Control \\
\hline Age & & & \\
\hline
\end{tabular}

Gender (male:female)

Hight

Body mass index $\left(\mathrm{kg} / \mathrm{m}^{2}\right)$

HRMAX

Score for self-reported

habitual physical activity

MoCA

BDI-II

\section{MMSE}

Behavioural tests for

cognitive ability (RT)

Behavioural test about

cognitive ability (ACC)

Behavioural tests for cognitive ability: Stroop task testing, n-back task testing, task-switch task testing.

ACC, accuracy; BDI-II, Beck Depression Inventory (2nd edition); HRMAX, maximum heart rate; MMSE, Mini-Mental State Examination; MoCA, Montreal Cognitive Assessment; RT, response time.

participants are recruited. They are double blind with recruitment and grouping.

\section{Exercise intervention}

The exercise intervention will last for 6 months. The participants will be divided into three groups: table tennis, fit aerobics and control. The table tennis (open-skill) and fit aerobics (closed-skill) groups will train for $60 \mathrm{~min}$, three times a week. The exercise intensity will be monitored, ensuring that the heart rate remains in the range of $60 \%-70 \%$ of the maximum heart rate (HRMAX $=220$ - age). During the training period, the exercise intensity will be monitored by the experimenter through real-time heart rate monitoring and the subject's subjective reaction. The exercise load and heart rate responses of the subjects will be recorded at each training session and adjusted accordingly with an increase of aerobic capacity. All the participants are not used to regular exercise before participating in the experiment. The control group just needs to maintain their old habits, and we will visit the control group regularly to evaluate their physical activity, so as to ensure that their physical activity does not meet the exercise standards. The entire intervention process will be monitored in real time by healthcare professionals. We will have coaches to monitor and record their attendance, and if they are absent, they will supplement the training at other times. Specific intervention methods are shown in table 2.

\section{SST protocol}

The SST was programmed using E-prime V.2, in which the subjects will be asked to press a " $\leftarrow$ " button or a " $\rightarrow$ " button according to the left and right arrow directions. In $25 \%$ of the trials, the arrow will turn red and a grey triangle will appear; the participants will press the button to stop or give no response. The interval of stimulation will be dynamically adjusted according to the tracking procedure. The task will be divided into four groups of 120 trials each. The task flow is shown in figure 2. The execution go signal reaction time (GoRT), stop response reaction time (SRRT), SSD and SSRT will be investigated. GoRT is how long it takes for the participant to respond after the GO (black arrow) appears. SRRT is the time to respond to the stop signal (arrow turning red) task. SSD is the time between the task stimulus and the stop signal.

The instructions for the experiment will be follows: Welcome to the experiment. First, "+" will appear in the centre of the screen to alert the user to begin the keystroke reaction, and the right index finger should be placed on the " $\downarrow$ " to prepare for the experiment. When the black arrow appears in the centre of the screen, use your right index finger to press the button. When the left arrow appears, use your index finger to press the " $\leftarrow$ " key on the keyboard. When the right arrow appears, use your index finger to press the " $\rightarrow$ " key on the keyboard. After each key response, the right index finger should quickly return to the " $\downarrow$ " key to wait for the next response. When the black arrow in the centre of the screen turns red and a small grey triangle appears, please stop the keystroke reaction immediately. When the red arrow appears in the centre of the screen, please do not perform any keystroke reaction. Let's move on to practice.

\section{OUTCOMES}

\section{Primary outcome}

Behavioral task

The task will include randomly interspersed no-go and stop-signal trials, which will enable us to examine both types of inhibition. A total of 480 trials will be performed, including $75 \%$ go trials and $25 \%$ stop trials. On go trials, the subjects will respond to a left/right black arrow (1000 ms) by pressing the buttons with their right hand. Responses will be made with the index finger (for the left or right arrow). In the stop-signal trials, a response will be initially cued by a left/right black arrow, but the arrow colour will change to red concurrent with a grey triangle after an SSD, and the subjects will be asked not to respond. The SSD will be varied from trial to trial by using a step-up/step-down algorithm with an initial estimate of $250 \mathrm{~ms}$ to maintain $50 \%$ successful inhibition. The task flow chart is shown in figure 3 .

The task will consist of randomly interspersed no-go and stop-signal trials. A total of 480 trials will be performed (75\% go, $25 \%$ stop). 
Table 2 Exercise intervention TIDieR

\begin{tabular}{|c|c|c|c|}
\hline \multirow{2}{*}{$\begin{array}{l}\text { Item } \\
\text { no. }\end{array}$} & \multirow[b]{2}{*}{ Brief name } & \multicolumn{2}{|l|}{ Group } \\
\hline & & Table tennis group & Fit aerobics group \\
\hline 1 & Why & Table tennis & Fit aerobics \\
\hline 2 & What & $\begin{array}{l}\text { Participants will be led by professional } \\
\text { table tennis coaches for } 6 \text { months of table } \\
\text { tennis exercise. }\end{array}$ & $\begin{array}{l}\text { Participants will be led by professional fit } \\
\text { aerobics coaches for } 6 \text { months of fit aerobics } \\
\text { exercise. }\end{array}$ \\
\hline
\end{tabular}

\begin{tabular}{|c|c|c|c|}
\hline 3 & What (materials) & \multicolumn{2}{|c|}{$\begin{array}{l}\text { Participants will do } 60 \mathrm{~min} \text { of exercise three times a week. Specific exercises will be } \\
\text { described later. }\end{array}$} \\
\hline 4 & $\begin{array}{l}\text { What } \\
\text { (procedures) }\end{array}$ & \multicolumn{2}{|c|}{$\begin{array}{l}\text { Each participant received a test report before and at } 3,6 \text { and } 12 \text { months after the start of } \\
\text { understand their physical and psychological changes. }\end{array}$} \\
\hline 5 & Who provided & $\begin{array}{l}\text { Table tennis coaches is the second } \\
\text { level table tennis athlete in China from } \\
\text { Shanghai University of Sport. The } \\
\text { psychology-related tests were completed } \\
\text { by Ph.D. students majoring in psychology } \\
\text { at Shanghai University of Sport. }\end{array}$ & $\begin{array}{l}\text { Fit aerobics coaches is the second level fit } \\
\text { aerobics athlete in China from Shanghai } \\
\text { University of Sport. The psychology-related } \\
\text { tests were completed by Ph.D. students } \\
\text { majoring in psychology at Shanghai } \\
\text { University of Sport. }\end{array}$ \\
\hline 6 & How & \multicolumn{2}{|c|}{$\begin{array}{l}\text { The exercise intervention will take place in a stationary gym, where the instructor will } \\
\text { direct the whole group face to face. }\end{array}$} \\
\hline
\end{tabular}

$7 \quad$ Where Table tennis training is held in China Table Tennis College of Shanghai Sport University. There is a professional table tennis training ground.

Fit aerobic trianing is conducted in the School of Physical Education and Training of Shanghai Sport University. There is a professional aerobics training ground.

8 When and how The table tennis (open-skill) and fit aerobics (closed-skill) groups will train for $60 \mathrm{~min}$, much three times a week. The exercise intensity will be monitored, ensuring that the heart rate remains in the range of $60 \%-70 \%$ of the maximum heart rate (HRMAX $=220-$ age).

Each session will consist of a warm-up The participants in the closed-skill exercise for $10 \mathrm{~min}$, table tennis practice under the guidance of a coach for $40 \mathrm{~min}$, and relaxation for $10 \mathrm{~min}$. The degree of difficulty of training throughout the programme will be gradually increased. Table tennis at $50 \%-60 \%$ of the maximum heart rate for the first 2 weeks, followed by $60 \%-70 \%$ of the maximum heart rate. The training will consist of the following seven main parts: (1) footwork; (2) the serve; (3) forehand backhand attack; (4) forehand backhand loop; (5) kill shot; (6) continuous hitting of directional or nondirectional balls randomly sent by the server; and (7) comprehensive practice. All the technical movements will be from simple to complex. or gymnastics to warm up and achieve a
The participants in the closed-skill exercise
group will attend a supervised fit aerobics class of the same frequency, duration, and length as the open-skill exercise group. Fit aerobics at $50 \%-60 \%$ of the maximum heart rate for the first 2 weeks, followed by $60 \%-70 \%$ of the maximum heart rate. Each fit aerobics class will consist of warm-up activities for $10 \mathrm{~min}$, basic aerobics activities for $40 \mathrm{~min}$, and relaxation and stretching for $10 \mathrm{~min}$. The fit aerobics training will involve the following: (1) jogging, rope skipping, and/ slightly sweating body, and the heart rate will be slightly faster; (2) stepping, side parallel step, side cross step, jumping jacks, lunging, kicking, and so on. Following the rhythm of the music, each action will be reduced from four sets to two sets and then to one set; (3) relax the shoulder, elbow, hip, and knee joints by doing a few stretches to help relax the muscles.

\section{Control group}

Control

Keep the original living habits, do not have regular exercise, regularly attend health lectures and accept telephone follow-up.

Participants will attend biweekly health talks and will be interviewed by phone once a month.

the exercise so that they could The health lecture was completed by Ph.D. students majoring in physical Education and training in Shanghai University of Sport, and the telephone return visit was completed by Ph.D. students majoring in psychology.

Health lectures will be conducted face to face in a group format, and telephone return visits will be conducted one by one.

The health lecture will be held in the conference room of school of Psychology, Shanghai University of Sport.

Fortnightly health lectures of $30-$ 50 min will be held. A 10 min return call once a month.

$\begin{array}{ll}9 \text { Tailoring } & \begin{array}{l}\text { Exercise interventions were performed at } 50 \%-60 \% \text { of maximum heart rate for the first } \\ 2 \text { weeks, followed by } 60 \%-70 \% \text { of maximum heart rate. In order to ensure the safety of } \\ \text { participants, the exercise intensity will be gradually increased, and the first } 2 \text { weeks will be } \\ \text { mainly acclimatisation. }\end{array} \\ 10 \text { How well } & \begin{array}{l}\text { Feedback will be given to each participant on a regular basis, including their physical and psychological data, as well as their } \\ \text { (planned) }\end{array} \\ \text { motor skills learning performance. Keep them up to date on their progress and status to keep them engaged. }\end{array}$

TIDieR, Template for Intervention Description and Replication.

\section{Secondary outcomes}

Secondary outcome measures will include the following: (1) Montreal Cognitive Assessment (MoCA); (2) Beck Depression Inventory (2nd edition) (BDI-II); (3) MiniMental State Examination (MMSE); (4) Stroop task testing; (5) n-back task testing; and (6) task-switch task testing (table 3). The process of inhibitory control is the process of inhibition, refresh and switching of the response task through cognitive function, so we will test the abilities of inhibition, refresh and switching in the 
go

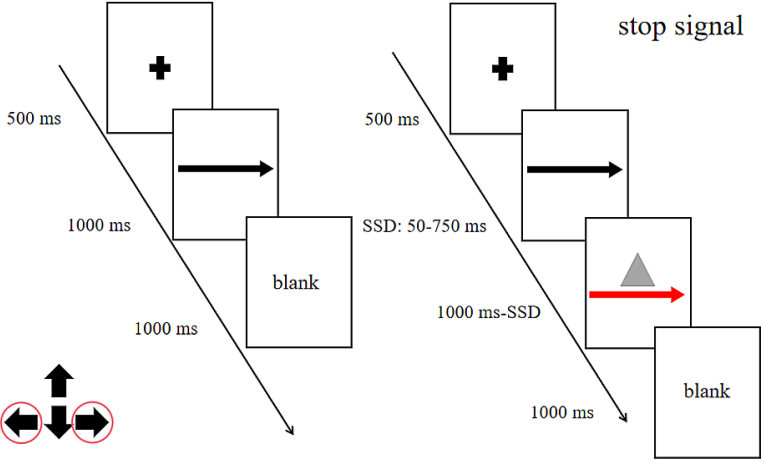

Figure 2 Stop signal task flow chart.

cognitive process of the elderly with different exercise types before and after the intervention by the Stroop task, the n-back task and the task-switch task to compare their response inhibitory ability. The details of each outcome and instrument will be compiled as in table 3 .

\section{Montreal Cognitive Assessment}

The MoCA is an assessment tool for the rapid screening of cognitive dysfunction. It includes 11 items in 8 cognitive domains: attention and concentration, executive function, memory, language, abstract thinking and computation. ${ }^{17}$ The highest possible total score is 30 , and a score of 26 or more is normal. It has a high sensitivity and a short test time, so it is suitable for clinical application.

\section{Beck Depression Inventory (2nd edition)}

The BDI-II contains 21 topics, which are suitable for people older than 13 years old. The areas of focus include symptoms of depression, such as despair, sensitivity, ways of understanding things and physical characteristics, such as fatigue, weight loss and decreased sexual ability. ${ }^{18}$

\section{Mini-Mental State Examination}

The MMSE Scale includes the following seven aspects: time orientation, place orientation, immediate memory, attention and calculation, delayed memory, language, and visual space ${ }^{19}$ There are a total of 30 questions asked in the exam, with 1 point awarded for each correct answer, and 0 points given for each wrong answer or unanswered question. The total score range of the scale is $0-30$ points. The test scores are closely related to the educational level of the subject. The normal cut-off values are as follows: illiterate subjects, >17; primary school education only, $>20$; junior high school education and above, $>24$.

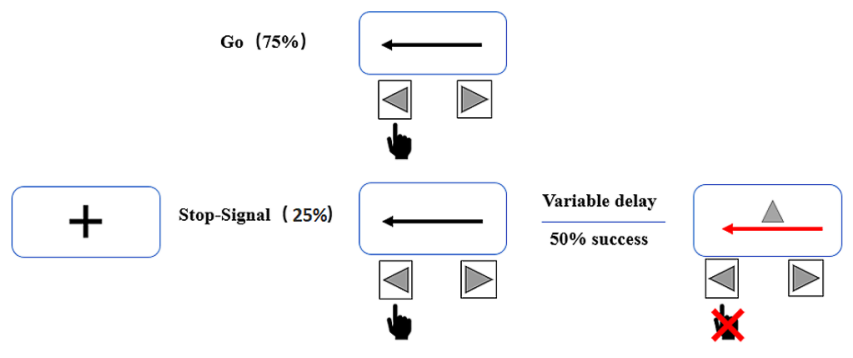

Figure 3 Stop behavioural task.
Stroop task

After a period of exercise intervention, comparison of the Stroop task response time between the open-skill, closed-skill, and control groups will be used to explore which types of exercise can improve cognitive inhibition ability. ${ }^{20}$

\section{$\mathrm{N}$-back task}

By comparing the n-back task accuracy of the elderly in the three groups, the improvement of the cognitive refresh ability of each group will be compared under different cognitive loads. ${ }^{21}$

\section{Task-switch task}

By comparing the task-switch accuracy of the three groups under different stimulus conditions, we will determine which type of exercise can improve the cognitive transformation ability most significantly. ${ }^{22}$

\section{Patient and public involvement}

Participants have not been involved in the study recruitment. The authors conceived the initial research questions and outcome measures, and modified according to the telephone interviews with patients and their guardians by a research assistant. In order to assure the safety and feasibility of the intervention, we invited six elderly peoples to learn and practise the table tennis and fit aerobics exercise before designing the RCT. Table tennis and fit aerobics exercise were revised based on the exercise performance and feedback provided by the participants. The burden of the intervention will be assessed by patients and their advisors through face-to-face interviews before signing informed consent. The findings of the study will be disseminated to the participants and their guardians.

\section{Statistical analysis}

The outcome assessor will be completed by the designated medical institution (Shanghai Punan Hospital of Pudong New District, Shanghai, China), when the participants go for the assessment, there will be no labels, so the outcome assessors will be blind in the group allocation. In order to ensure no intergroup differences in all test indicators after random grouping, Independent sample t-test will be performed on each pretest data after random grouping. Repeated measures analysis of variance will be used to determine the effects of different types of exercise (open, closed or control) on the primary outcomes (GoRT, SRRT, SSD and SSRT) as well as on the secondary outcomes (MoCA Score, BDI-II Score, MMSE Score, Stroop response time, n-back accuracy, task-switch accuracy), with group and time-serving as factors. Data will be analysed by Ph.D. students who are blind to group allocation.

\section{DISCUSSION}

Studies have found that elderly people who participate in ball and racket projects have higher health and physical awareness. ${ }^{23}$ Pilates exercises decreased depression and 
Table 3 The differences among the three groups of subjects at various stages

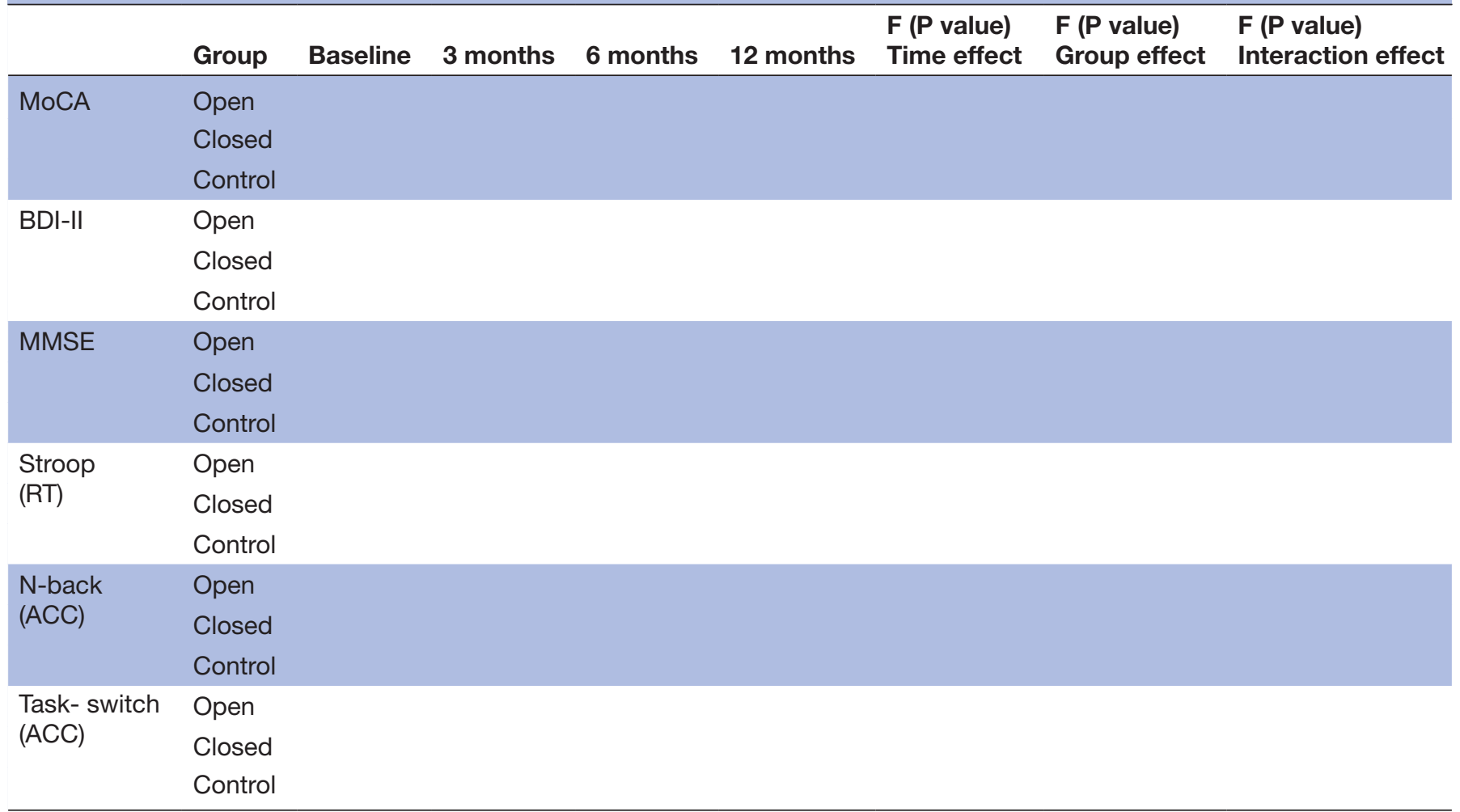

ACC, accuracy; BDI-II, Beck Depression Inventory (2nd edition); MMSE, Mini-Mental State Examination; MoCA, Montreal Cognitive Assessment; RT, response time.

improved the balance related to falling in elderly, ${ }^{24}$ and also experienced significant improvement in physical, social spiritual and emotional wellness. ${ }^{25}$ Korean scholars found the cognitive/exercise dual-task programme was an effective intervention for improving cognitive function, health status and life satisfaction, and for decreasing depression of the elderly living in the community. ${ }^{24}$

Then some researchers found that athletes participating in open-skill exercises have a faster switching speed, fewer cognitive resources and a higher efficiency in the inhibition process. ${ }^{26}$ At the same time, another study has demonstrated that athletes who engage in open-skill exercises have a stronger reaction switching capability. ${ }^{27}$ Based on previous research, participating in sports involving open-skill exercises can improve the ability of inhibition, refresh and conversion in the inhibitory control process. In the Stoop task, when two different dimensions of a stimulus interfere with each other, it is necessary for participants to inhibit the interfering stimulus, it looked at the participants' ability to suppress interference. In the n-back task, participants' attention capacity and memory capacity could be examined during the process of stimulus updating. In task-switch task, participants need to keep their attention focused when switching between different tasks, so it can investigate the transformation ability of participants in the cognitive process. Therefore, in this study, the Stroop task, n-back task, and task-switch task were selected as secondary outcomes to investigate the inhibition, refresh and transformation abilities, respectively, of the participants. Determining the effect of long-term intervention of different types of sports on the response inhibitory ability of the elderly will improve our understanding of inhibitory control degradation and support the development of diversified training programmes.

This study will use the SST to explore how different types of exercise experience affect the response inhibition ability in the elderly. Because of the interactions of the cognitive level, depressive state and mental state with the response inhibitory ability, we will perform multiple MoCA, BDI-II, MMSE measurements before and after each intervention. We will also explore whether sports involving open-skill exercises can improve the cognitive level, depression state and mental state of the elderly while improving their inhibition and control ability. Through multidimensional measurements of the participants, we hope to provide a more sufficient theoretical basis for the mechanism of improving the inhibition and control ability of the elderly through exercise.

This study will have several limitations. In this research, the screening principles of the subjects will be determined in the longitudinal study, and the inclusion criteria and exclusion criteria will be strictly defined, especially the experimental groups and the control group in terms of age, physical level, intervention time and intensity. In addition, because the exercise that will serve as an 
intervention will be widely known to the participants, the single-blind design may expose the study to risks of bias stemming from performance and evaluation, which may potentially lead to overestimation of the effects of exercise.

\section{ETHICS AND DISSEMINATION}

All participants who meet the inclusion and exclusion criteria will be required to sign an informed consent form prior to enrolling in the study. This study was approved by the Shanghai University of Sport Research Ethics Committee (102772019RT012). Study findings will be disseminated via publications in peer-reviewed journals and presentations at international conferences.

Contributors LK: Conceptualisation, methodology and software. ZL: Writingreviewing and editing. ZJ: Visualisation and investigation. WJ: Data curation and writing —original draft preparation.

Funding This study was supported by grants from the outstanding clinical discipline project of Shanghai Pudong (PWYGy 2018-04) and National Natural Science Foundation of China (3197070657).

Competing interests None declared.

Patient and public involvement Patients and/or the public were involved in the design, or conduct, or reporting, or dissemination plans of this research. Refer to the Outcomes section for further details.

Patient consent for publication Not applicable.

Provenance and peer review Not commissioned; externally peer reviewed.

Open access This is an open access article distributed in accordance with the Creative Commons Attribution Non Commercial (CC BY-NC 4.0) license, which permits others to distribute, remix, adapt, build upon this work non-commercially, and license their derivative works on different terms, provided the original work is properly cited, appropriate credit is given, any changes made indicated, and the use is non-commercial. See: http://creativecommons.org/licenses/by-nc/4.0/.

ORCID iD

Wei Jianing http://orcid.org/0000-0002-0973-7661

\section{REFERENCES}

1 Logan GD, Van Zandt T, Verbruggen F, et al. On the ability to inhibit thought and action: general and special theories of an act of control. Psychol Rev 2014;121:66-95.

2 Darowski ES, Helder E, Zacks RT, et al. Age-Related differences in cognition: the role of distraction control. Neuropsychology 2008;22:638-44.

3 Chen TY. Cognitive aging and executive decline hypothesis. Advances in Psychological Science 2004;05:729-36.

4 Craik FIM, Bialystok E, Fergus IM. Cognition through the lifespan: mechanisms of change. Trends Cogn Sci 2006;10:131-8.
5 Timothy A. Aging and measures of processing speed. Biol Psychol 2000;54.

6 Zhang NN. Effect of long-term tai chi exercise on cognitive function of middle-aged and old people. Chinese $J$ Rehabilitation Med 2006;26:7-9.

7 Wang C-H, Chang C-C, Liang Y-M, et al. Open vs. closed skill sports and the modulation of inhibitory control. PLoS One 2013;8:e55773.

8 Voss MW, Kramer AF, Basak C, et al. Are expert athletes 'expert' in the cognitive laboratory? A meta-analytic review of cognition and sport expertise. Appl Cogn Psychol 2010;24:812-26.

9 Logan GD, Cowan WB, Davis KA. On the ability to inhibit simple and choice reaction time responses: a model and a method. $J$ Exp Psychol 1984;10:276-91.

10 Smith JL, Jamadar S, Provost AL, et al. Motor and non-motor inhibition in the Go/NoGo task: an Erp and fMRI study. Int $J$ Psychophysiol 2013;87:244-53.

11 Verbruggen F, Logan GD, Gordon D. Response inhibition in the stopsignal paradigm. Trends Cogn Sci 2008;12:418-24.

12 Verbruggen F, Aron AR, Band GP, et al. A consensus guide to capturing the ability to inhibit actions and impulsive behaviors in the stop-signal task. Elife 2019;8. doi:10.7554/eLife.46323. [Epub ahead of print: 2904 2019].

13 Verbruggen F, Chambers CD, Logan GD. Fictitious inhibitory differences: how skewness and slowing distort the estimation of stopping latencies. Psychol Sci 2013;24:352-62.

14 Friehs MA, Frings C. Pimping inhibition: anodal tDCS enhances stop-signal reaction time. J Exp Psychol Hum Percept Perform 2018;44:1933-45.

15 Friehs MA, Frings C, Maximilian A. Cathodal tDCS increases stopsignal reaction time. Cogn Affect Behav Neurosci 2019;19:1129-42.

16 Faul F, ErdFelder E, Lang A-G. G*Power 3.1 manual. Behav Res Methods 2007;39:175-91.

17 Gao F. The application of MoCA test and DTI in mild cognitive impairment. Diss. MA thesis. PLA Military Medical Training College, 2008.

18 Holländare F, Andersson G, Engström I. A comparison of psychometric properties between Internet and paper versions of two depression instruments (BDI-II and MADRS-S) administered to clinic patients. J Med Internet Res 2010;12:e49-79.

19 Azmeh SMD. Mini-Mental state examination (MMSE). New York: Springer, 2011.

20 Chen J, Haiyan L, Jijia Z, et al. The latest advances of the Stroop Effect-its theroy, paradigms, affecting factors. J Psychol Sci 2007;02:415-8.

21 Song HK. The evolution and application of N-back paradigm. Journal of Southwest University 2011;37:81-2.

22 Shi Y, Zhou X. Task switching, a paradigm in the study of executive control. Advances in Psychological Science 2004;05:672-9.

23 Ahn C, Lee W. The difference between exercise effect, health and fitness perception according to the degree of exercise participation of the elderly. Journal of adapted physical activity and exercise 2021;29:245-54.

24 Mokhtari M, Nezakatalhossaini M, Esfarjani F. The effect of 12-week Pilates exercises on depression and balance associated with falling in the elderly. Procedia Soc Behav Sci 2013;70:1714-23.

25 Yeon RS. The effect of 12-week Pilates exercises on wellness in the elderly. J Exerc Rehabil 2016;12.

26 Di Russo F, Bultrini A, Brunelli S, et al. Benefits of sports participation for executive function in disabled athletes. J Neurotrauma 2010;27:2309-19.

27 Krenn B, Finkenzeller T, Würth S, et al. Sport type determines differences in executive functions in elite athletes. Psychol Sport Exerc 2018;38:72-9. 